\title{
A PILOT STUDY OF MATRIX METALLOPROTEINASES ON THE MODEL OF DAUNORUBICIN-INDUCED CARDIOMYOPATHY IN RABBITS
}

\author{
Anna Potáčová ${ }^{1}$, Michaela Adamcová ${ }^{1}$, Martin Štěrba ${ }^{2}$, Olga Popelová $^{2}$, Tomáš Šimůnek ${ }^{4}$, Yvona Mazurováa ${ }^{3}$, \\ Ivana Gunčová, ${ }^{3}$, Vladimír Geršl ${ }^{2}$
}

Charles University in Prague, Faculty of Medicine and University Hospital in Hradec Králové, Czech Republic: Department of Physiology ${ }^{1}$, Department of Pharmacology ${ }^{2}$, Department of Histology and Embryology ${ }^{3}$; Charles University in Prague, Faculty of Pharmacy in Hradec Králové, Czech Republic: Department of Biochemical Sciences ${ }^{4}$

Summary: Matrix metalloproteinases (MMPs), activated by oxidative stress, play a key role during cardiac remodeling. In the present study we aimed to assess the role of MMPs in experimental cardiomyopathy induced by repeated 10 -week administration of daunorubicin $(3 \mathrm{mg} / \mathrm{kg}$ i.v.) to rabbits. In the daunorubicin group, the plasma cardiac troponin $\mathrm{T}$ levels (cTnT - a marker of myocardial necrosis) were significantly increased $(\mathrm{p}<0.05)$, commencing with the $8^{\text {th }}$ administration compared with the controls. The amount of collagen (an estimate of fibrosis) was also significantly higher in the daunorubicin group $(13.39 \pm 0.97 \mathrm{mg} / \mathrm{g}$ wet weight) compared to the control group $(10.03 \pm 0.65 \mathrm{mg} / \mathrm{g}$ wet weight $)$. In both groups, the LV MMP-activity was observed only in the gelatine substrate in the $70 \mathrm{kDa}$ region (MMP-2), while no MMPs activities were detectable either in the casein or collagen containing zymograms. At the end of the experiment, the MMP2 activity was slightly up-regulated (by $16 \%$ ) compared with the controls.

Key words: Matrix metalloproteinases; Anthracycline; Daunorubicin cardiomyopathy; Remodelling; Collagen

An anthracycline-induced myocardial injury is typically characterized by progressive myofibrillar loss and degeneration of the left ventricular (LV) cardiomyocytes, ultimately resulting in dilated cardiomyopathy and congestive heart failure accompanied by both systolic and diastolic dysfunctions (2). The repeated long-term administration of daunorubicin to rabbits induces chronic cardiotoxicity and thus this rabbit model is considered to be a clinically relevant animal model for the study of anthracycline-induced cardiomyopathy (7). The principal role in the pathogenesis of this toxicity is attributed to reactive oxygen species generated by "redox-cycling" of anthracycline molecule and/or by the formation of anthracycline-ferric iron complexes (3). The death of cardiac cells triggered by oxidative stress reportedly involves both necrosis and apoptosis. The collagen fibrils subsequently replace degenerated myocytes. It is believed that activation of MMPs and reduction of abundance of tissue inhibitors of MMPs (triggered predominantly by reactive oxygen species) present the initial step of remodeling the extracellular matrix during adaptive response of the heart to the toxicity of anthracyclines. It brings about a destruction of some collagen links, with further increased synthesis of collagen type III and I. The significant alteration of the collagen network creates the basis for the development of the fibrosis accompanying the dilated cardiomyopathy (4).
So far, altering the activity of matrix metalloproteinases (MMPs) has been shown in an acute experimental setting only. Bai et al. (1) demonstrated the time-dependent activation of MMPs (12 h, 1-4 days) in the heart and in the aortic tissue after a single high dose injection of doxorubicin $(25 \mathrm{mg} / \mathrm{kg}$ i.p. $)$ in mice with an earlier onset in the aorta. Similarly, Pacher et al. (5) found that doxorubicin induced MMPs activity in the heart in a similar model (5 days). However, unlike chronic and delayed types of anthracycline cardiotoxicity, the acute toxicity induced by the single supratherapeutical dose is not a common clinical problem. Therefore we aimed to study, for the first time, the contribution of MMPs to the cardiac remodeling on the validated model of anthracycline-induced chronic cardiotoxicity and the methodological aspects of MMP activity determination using zymography.

All experiments used in this study were approved by the Ethical Committee of the Charles University, Faculty of Medicine in Hradec Králové. The study was carried out on two groups of Chinchilla male rabbits:

- control (saline $1 \mathrm{~mL} / \mathrm{kg}$ i.v.), once a week, 10 administrations, $\mathrm{n}=8$

- daunorubicin (Cérubidine, Bellon Rhone-Poulenc, France, $3 \mathrm{mg} / \mathrm{kg}$ i.v.), once a week, 10 administrations, $\mathrm{n}=11$

Concentrations of cardiac troponin $\mathrm{T}$ (cTnT, a marker of necrosis) in heparinized plasma samples were measured 
using Elecsys Troponin T STAT Immunoassay (Roche) on the Elecsys 2010 immunoassay analyser (Roche). Hydroxyproline (estimate of fibrosis) in myocardial tissue was determined spectrophotometrically according to the method of Reddy (6) and the factor of 7.46 was used to convert the total concentration of hydroxyproline to the concentration

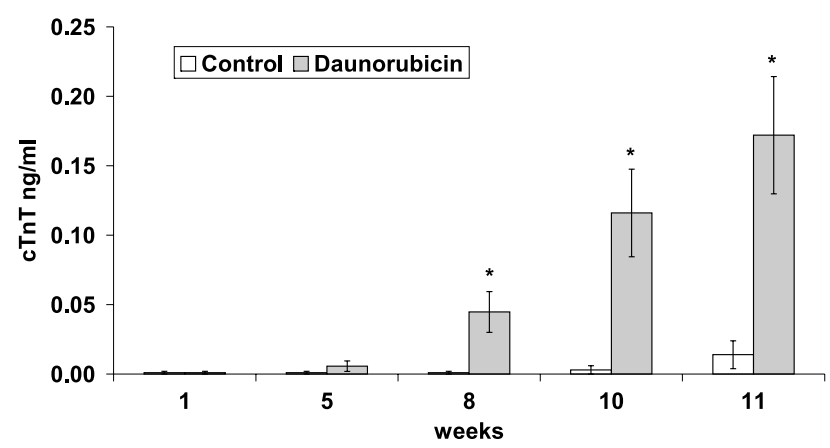

Fig. 1: Cardiac troponin $\mathrm{T}(\mathrm{cTnT})$ plasma concentrations in the model of chronic anthracyline-induced cardiomyopathy in rabbits. * - Statistical significance against control group (ANOVA; $\mathrm{P}<0.05$ ).

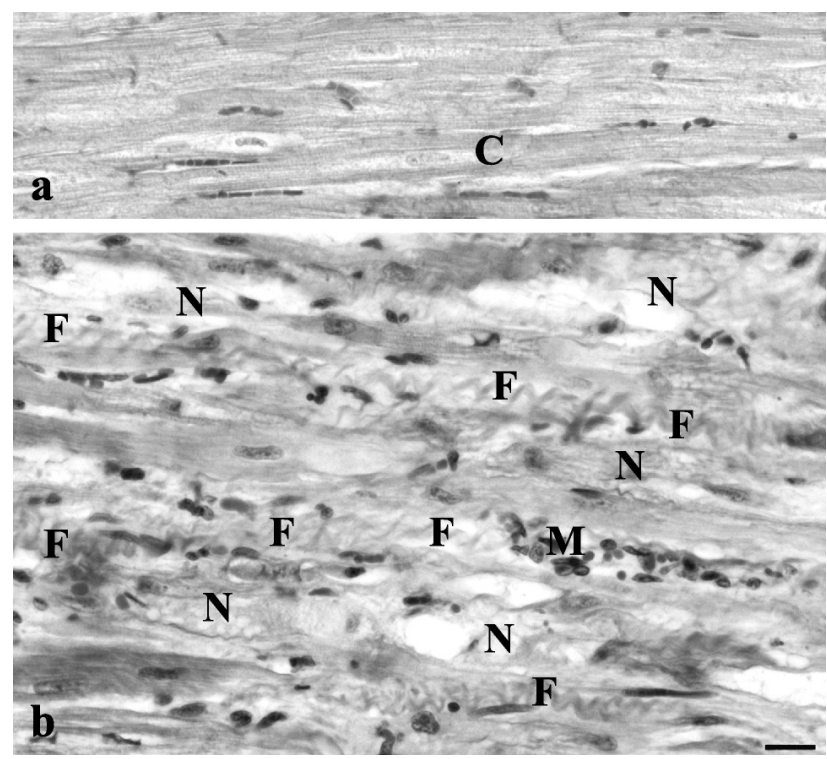

Fig. 2: Left ventricle myocardium of the rabbit heart. The myocardium reveals intensive toxic damage after protracted administration of daunorubicin, resulting in conspicuous myofibrosis (b) in comparison with the intact heart of control animals (a).

(a) Normal appearance of cardiomyocytes (C). (b) Degenerated and subsequently necrotic cardiomyocytes $(\mathrm{N})$ are removed by macrophages $(\mathrm{M})$ - which are already in a small number at this stage of damage - and gradually replaced by proliferated connective tissue marked by thick wavy collagen fibers $(F)$. Bundles of these fibers form small scars which later here and there become larger and denser. $\mathrm{a}, \mathrm{b}$ - Masson's blue trichrome; Bar $20 \mu \mathrm{m}$. of collagen. MMP enzyme activities were assayed by SDSPAGE zymography using either gelatine (Type A, SigmaAldrich), casein ( $\beta$-casein, Sigma-Aldrich) or collagen (Collagen Type I, Sigma-Aldrich) as MMP substrates. Enzyme activities attributed to MMPs can be visualized in the $\mathrm{zy}$ mograms as clear bands against a blue background of undegraded substance, and their relative molecular weights were estimated by comparison with the migration of standards (Chemicon, USA). Relative clearing of each sample was quantified by determining the inverse optical density units using GS-800 Calibrated Densitometer (Bio-Rad, USA) with Quantity One software. An histological examination after autopsy was performed using light microscopy.

In the daunorubicin group, premature mortality (18.2\%) was observed. During the autopsy, which was always performed immediately after the death, signs of heavy congestion were obvious - pleural effusion and ascites were present. Since myocardial samples in both prematurely dead animals were appropriately obtained, these were also taken into the present analysis. In the daunorubicin group, plasma cTnT levels were significantly increased $(\mathrm{p}<0.05)$ commencing with the $8^{\text {th }}$ administration, while in the control group, the plasma cTnT levels were below $0.02 \mu \mathrm{g} / \mathrm{L}$ during the whole experiment - Fig. 1. The total amount of collagen in myocardial samples was significantly higher in the daunorubicin group (13.39 $\pm 0.97 \mathrm{mg} / \mathrm{g}$ wet weight $)$ as compared with the control group $(10.03 \pm 0.65 \mathrm{mg} / \mathrm{g}$ wet weight). The histological examination (on paraffin sections) revealed the normal appearance of the myocardium in the control group - increased eosinophilia of cytoplasm was present in some cardiomyocytes, wheareas signs myofibrillar degeneration were seen only sporadically. On the other hand, the typical morphological picture of focal toxic damage of the myocardium was observed in the daunorubicin group, followed by reparative changes resulting in interstitial fibrosis - Fig. 2.

To study the role of MMPs in the development of anthracycline cardiotoxicity, we have investigated the activity of MMP using zymography, with all available substrates preferred by particular type of MMP. Zymography with gelatine (which is the only substrate employed in most of the studies published so far) is mainly used for the detection of gelatinases, i.e., MMP-2 and MMP-9. Unfortunately, casein zymography is less sensitive and casein migrates in the gel

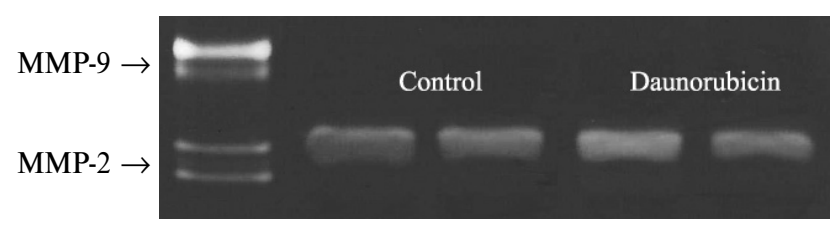

Fig. 3: Representative gelatinase zymography showing the MMP-activity at the end of experiment (11 weeks) on the validated model of daunorubicin-induced cardiomyopathy in rabbits in the comparison with the migration of standards MMP-2/MMP-9 (Chemicon, USA). 
during electrophoresis due to its relatively low molecular weight $(23 \mathrm{kDa})$. To reduce the anodic migration, it is first necessary to pre-run the casein gel to allow the excess of casein to migrate out. A second run of the gel does not cause any further migration of the casein, thus distributing casein in the gel uniformly and eliminating its interference with MMP bands. The incorporation of native collagen fibers in polyacrylamide gels appears unsuitable for zymography because of their complicated structure, but if collagen type I (Sigma-Aldrich) is used, SDS disrupts most of the fibrillar organization of the collagen, allowing proteins to run into the gel.

After the successful resolvement of the above mentioned methodological issues, it was possible to appropriately evaluate the MMPs activity in the left ventricular myocardial samples obtained from rabbits. In the control group, the proteolytic activity was observed only in the gelatine substrate in the $72 \mathrm{kDa}$ region corresponding to the gelatinase A, MMP-2. MMP-2 activity was slightly upregulated in the daunorubicin group (by $\approx 16 \%$ ) - Fig. 3, which also involved animals with premature death $(18.2 \%)$. No MMPs-activities were detectable either in the casein- or collagen-containing zymograms.

Although the involvement of metalloproteinases in the development of anthracycline-induced cardiomyopathy was suggested in literature, the experimental data are scarce. In general, three distinct phases of cardiac remodelling appear during heart failure (4). In the initial phase, fibrillar collagen is partially degraded due to increased matrix metalloproteinase activity. Following this, there is a chronic compensatory phase during which MMP activity and collagen concentration return to normal. The final phase is characterized by elevated MMP activity, marked ventricular dilatation and prominent fibrosis. Concomitantly, Janicki (4) emphasized that the temporal pattern of MMPs activation can be unique to the type of pathological stimulus.
In conclusion, we demonstrated that in our model of anthracycline cardiomyopathy in rabbits in vivo only MMP2 activity is slightly up-regulated after long-term administration of daunorubicin in the $11^{\text {th }}$ week, while the activities of other MMP were undetectable by zymography (it is also possible that these enzymes do not contribute to this process at all). As the MMP activity can undergo dramatic changes in the course of anthracycline cardiotoxicity, it is plausible that more significant changes can be present in earlier phases. Hence, further studies are needed for assessment of the role of MMPs in the development of anthracycline cardiotoxicity.

\section{Acknowledgments:}

The authors wish to thank Mrs. Ludmila Koželuhová for her technical assistance.

This study was supported by the Charles University Grant Agency No. 7531/2007 C.

\section{References:}

1. Bai P, Mabley JG, Liaudet L, Virág L, Szabó C, Pacher P. Matrix metalloproteinase activation is an early ebeny in doxorubicin-induced cardiotoxicity. Oncol Rep. 2004;11:505-8.

2. Herman EH, Zhang J, Hasinoff BB, Clark JR Jr, Ferrans VJ. Comparison of the structural changes induced by doxorubicin and mitoxantrone in the heart, kidney and intestine and characterization of the $\mathrm{Fe}(\mathrm{III})$-mitoxantrone complex. J Mol Cell Cardiol. 1997;29(9):2415-30.

3. Hrdina R, Geršl V, Klimtová I, Šimůnek T, Macháčková J, Adamcová M. Anthracycline-induced cardiotoxicity. Acta Medica (Hradec Kralove). 2000;43(3):75-82.

4. Janicki JS, Brower GL, Gardner JD et al. The dynamic interaction between matrix metalloproteinase activity and adverse myocardial remodeling. Heart Fail Rev 2004;9:33-42.

5. Pacher P, Liaudet L, Bai P, Virag L, Mabley JG, Hasko G, Szabo C. Activation of poly(ADP-ribose) polymerase contributes to development of doxorubicin-induced heart failure. J Pharmacol Exp Ther. 2002;300(3):862-7.

6. Reddy GK and Enwemeka CS. A simplified method for the analysis of hydroxyproline in biological tissues. Clin Biochem 1996;29:225-29.

7. Šimůnek T, Klimtová I, Kaplanová J, Mazurová Y, Adamcová M, Štěrba M, Hrdina R, Geršl V. Rabbit model for in vivo study of anthracycline-induced heart failure and for the evaluation of protective agents. Eur J Heart Failure 2004; 6:377-387.

\section{Corresponding author:}

Mgr. Anna Potáčová, Charles University in Prague, Faculty of Medicine in Hradec Králové, Department of Physiology, Šimkova 870, 50038 Hradec Králové, Czech Republic; e-mail: potacovaa@lfhk.cuni.cz 